\title{
Optimization of Power Consumption in Wireless Access Networks Using Differential Evolution with Eigenvector Based Crossover Operator
}

\author{
Sotirios K. Goudos ${ }^{1}$, Margot Deruyck ${ }^{2}$, David Plets ${ }^{2}$, Luc Martens ${ }^{2}$, Wout Joseph ${ }^{2}$ \\ ${ }^{1}$ Radiocommunications Laboratory, Department of Physics, Aristotle University of Thessaloniki, Thessaloniki, Greece \\ ${ }^{2}$ bWiCa, Ghent University / iMinds, Dept. of Information Technology, Gaston Crommenlaan-8-box-201, B-9050 Ghent, Belgium
}

\begin{abstract}
The power consumption of wireless access networks is an important issue. In this paper, the power consumption of LTE base stations is optimized. We consider the city of Ghent, Belgium with 75 possible LTE base station locations. We optimize the network towards two objectives: the coverage maximization and the power consumption minimization. We propose a design framework based on Differential Evolution with eigenvector-based crossover operator. The results of the proposed method indicate the advantages and applicability of our approach.
\end{abstract}

Index Terms-power consumption, base station, coverage, LTE, differential evolution, network planning.

\section{INTRODUCTION}

Wireless access networks are currently large power consumers within ICT. In five years' time (from 2007 till 2012 ), this power consumption has increased yearly with $10 \%$ [1]. It is expected that this amount will even increase in the next few years, as these networks need to expand in order to deal with the extreme growth of mobile devices and the higher bit rate demands required by these mobile devices. For the development of future wireless access networks, power consumption will become a key parameter [2, 3]. A specified area, the target area, needs to be covered with a certain wireless technology. In this paper we consider Long Term Evolution (LTE) with a minimal power consumption. By selecting the most appropriate base station locations from a set of existing locations (from operators active in the target area) and tuning base station parameters such as the antenna's input power, an energy-efficient network is obtained. Evolutionary algorithms (EAs) are suitable optimization techniques for solving the above-described problem. The purpose of this paper is to solve this problem using a recently introduced Differential Evolution (DE) [4] variant.

DE is a population-based stochastic global optimization algorithm that has been applied to a variety of design problems in electromagnetics. Several DE variants or strategies exist. One of the DE advantages is that very few control parameters have to be adjusted in each algorithm run. The DE performance depends on control parameters, mutation strategies, and crossover operators. Most of the DE strategies or variants use the binomial crossover operator, which has been found to produce better results than the exponential crossover operator [5]. The authors in [6] propose an alternative crossover operator, namely the eigenvector-based crossover. This operator utilizes the eigenvector information of the covariance matrix of the population to rotate the coordinate system. In this paper, we apply the DE-EIG to the LTE network-planning problem. More specifically, we utilize the DE-EIG algorithm for designing different LTE networks cases that maximize coverage and reduce power consumption.

This paper is organized as follows. We describe the problem formulation in Section II. The details of the DE-EIG algorithms are given in Section III. In Section IV we present the numerical results. Finally, the conclusion is given in Section V.

\section{PRoblem Definition}

We consider 75 possible LTE base stations locations in the city of Ghent, Belgium to which the network planning optimization will be applied (Fig. 1). This area covers about $6.85 \mathrm{~km}^{2}$.

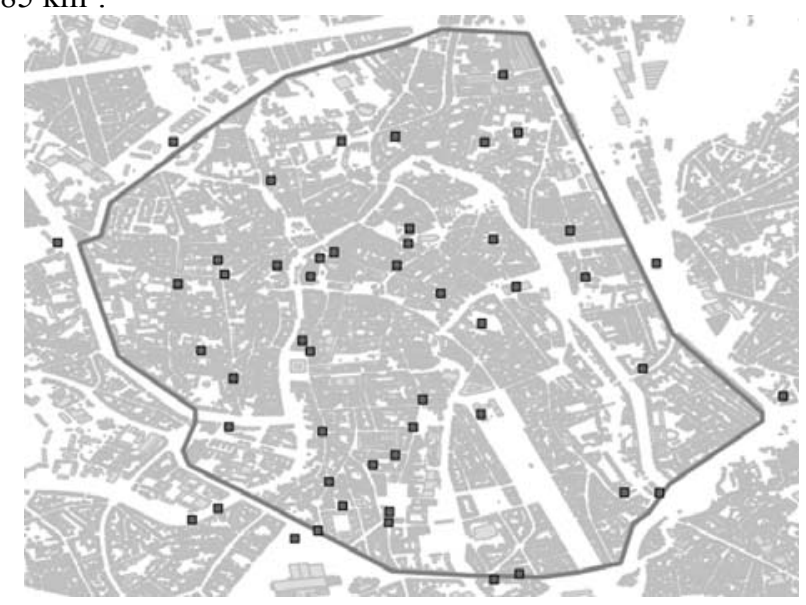

Fig. 1. Map of the city of Ghent, Belgium with the possible LTE base stations locations.

The network optimization problem is to find the least possible number of base stations that operate with such input power so that the coverage area is maximized. Therefore, there are two requirements; to minimize power consumption and to maximize coverage. The power consumption objective 
can be expressed as [7]:

$$
f_{\text {pow }}(\bar{x})=100\left(1-\frac{P(\bar{x})}{P_{\max }}\right)
$$

where $\bar{x}$ is the vector of a given solution, $P(\bar{x})$ is the power consumption in Watts of the solution, and $P_{\max }$ is the maximum power consumption assuming that all base station are active and operate at maximum input power. The details of the power consumption formulation can be found in [7].

The second objective is to cover the maximum possible percentage of the given area (Ghent in this case). This is given by:

$$
f_{\text {cov }}(\bar{x})=100 \frac{A_{\text {target }} \cap A(\bar{x})}{A_{\text {target }}}
$$

where $A_{\text {target }}$ is the area of the target area to be covered (in $\left.\mathrm{km}^{2}\right), A(\bar{x})$ is the area covered by a given solution (in $\mathrm{km}^{2}$ ), and $\bigcap$ represents the intersection of the two areas. In order to calculate the $A(\bar{x})$ we need first to calculate for each active base station the maximum allowable path loss, PLmax (in $\mathrm{dB})$. For this case, the link budget parameters for the LTE network of Table 1 are taken into account. Then the maximum range $R$ (in meters) covered by each base station can be computed [7]. The area covered by a given solution is the union of all base stations coverage areas that are determined by each maximum range $R$.

The above objectives can be combined using the following objective function [7]:

$$
F(\bar{x})=-\left(f_{\text {cov }}(\bar{x})+k f_{\text {pow }}(\bar{x})\right)
$$

with

$$
k=\left\{\begin{array}{l}
0 \text { if } f_{\mathrm{cov}}(\bar{x})<90 \\
\frac{\left(f_{\mathrm{cov}}(\bar{x})-90\right)^{2}}{5} \text { if } 90 \leq f_{\mathrm{cov}}(\bar{x}) \geq 95 \\
5 \text { otherwise }
\end{array}\right.
$$

where the minus sign is used for minimization. The minimum value (-600) is obtained when both $f_{\text {cov }}(\bar{x})$ and $f_{\text {pow }}(\bar{x})$ equal to 100 . This kind of global fitness function is chosen because of the trade-off between coverage and power consumption.

In this paper, we consider the Walfisch-Ikegami propagation model for path loss calculations. The abovementioned problem can be solved using an evolutionary algorithm. It is an integer-programming problem, for which

\begin{tabular}{|c|c|}
\hline Parameter & Value \\
\hline Frequency & $2.6 \mathrm{GHz}$ \\
\hline $\begin{array}{l}\text { Maximum input power base station } \\
\text { antenna }\end{array}$ & $43 \mathrm{dBm}$ \\
\hline Antenna gain of base station & $18 \mathrm{dBi}$ \\
\hline Antenna gain of receiver & $0 \mathrm{dBi}$ \\
\hline Feeder loss base station & $2 \mathrm{~dB}$ \\
\hline Feeder loss receiver & $0 \mathrm{~dB}$ \\
\hline Fade margin & $10 \mathrm{~dB}$ \\
\hline Yearly availability & $99.995 \%$ \\
\hline Interference margin & $2 \mathrm{~dB}$ \\
\hline Noise figure of receiver & $8 \mathrm{~dB}$ \\
\hline Implementation loss of receiver & $0 \mathrm{~dB}$ \\
\hline MIMO & $1 \times 1$ \\
\hline Receiver SNR & $\begin{array}{c}1 / 3 \mathrm{QPSK}=-1.5 \mathrm{~dB} \\
1 / 2 \mathrm{QPSK}=3 \mathrm{~dB} \\
2 / 3 \mathrm{QPSK}=10.5 \mathrm{~dB} \\
1 / 216-\mathrm{QAM}=14 \mathrm{~dB} \\
2 / 316-\mathrm{QAM}=19 \mathrm{~dB} \\
1 / 264-\mathrm{QAM}=23 \mathrm{~dB} \\
2 / 364-\mathrm{QAM}=29.4 \mathrm{~dB}\end{array}$ \\
\hline Bandwidth & $5 \mathrm{MHz}$ \\
\hline Soft handover gain receiver & $0 \mathrm{~dB}$ \\
\hline Building penetration loss & $\begin{array}{l}0 \mathrm{~dB} \text { (only outdoor } \\
\text { coverage considered) }\end{array}$ \\
\hline Height mobile station & $1.5 \mathrm{~m}$ \\
\hline
\end{tabular}
several different solutions exist. In this paper, we will apply the DE-EIG algorithm.
TABLE I. LINK BUDGET PARAMETERS FOR THE LTE NETWORK

\section{DIFFERENTIAL EVOLUTION WITH EIGENVECTOR-BASED} CROSSOVER OPERATOR (DE-EIG)

A population in DE consists of $N P$ vectors $\bar{x}_{G, i}, i=1,2, \ldots \ldots . N P$, where $G$ is the generation number. The population is initialized randomly from a uniform distribution. Each $D$-dimensional vector represents a possible solution, which is expressed as:

$$
\bar{x}_{G, i}=\left(x_{G, 1 i}, x_{G, 2 i}, \ldots x_{G, j i}, \ldots . ., x_{G, D i}\right)
$$

The population is initialized as follows: $x_{0, j i}=\operatorname{rand}_{j[0,1)}\left(x_{j, U}-x_{j, L}\right)+x_{j, L} \quad j=1,2, \ldots \ldots, D(5)$

where $x_{j, L}$ and $x_{j, U}$ are the lower and upper bounds of the jth dimension respectively and $\operatorname{rand}_{j[0,1)}$ is a uniformly distributed random number within $[0,1)$.

The initial population evolves in each generation with the use of three operators: mutation, crossover, and selection. Depending on the form of these operators, several DE variants or strategies exist in the literature $[4,8]$. The choice of the best DE strategy depends on problem type [5]. A mutant vector $\bar{v}_{G+1, i}$ is generated using

$$
\bar{v}_{G+1, i}=\bar{x}_{G, r_{1}}+F\left(\bar{x}_{G, r_{2}}-\bar{x}_{G, r_{3}}\right), \quad r_{1} \neq r_{2} \neq r_{3}
$$

where $r_{1}, r_{2}, r_{3}$ are randomly chosen indices from the population, which are different from index $i, F$ is a mutation control parameter, and $\operatorname{rand}_{[0,1)}$ is a randomly generated number from a uniform distribution within the interval $[0,1)$. 
In the popular $D E / \mathrm{rand} / 1 / \mathrm{bin}$ strategy the binary crossover operator is applied to generate a trial vector $\bar{u}_{G+1, i}$ whose coordinates are given by:

$u_{G+1, j i}= \begin{cases}v_{G+1, j i}, & \text { if } \operatorname{rand}_{j[0,1)} \leq C R \text { or } \mathrm{j}=\mathrm{rn}(\mathrm{i}) \\ x_{G, j i}, & \text { if } \operatorname{rand}_{j[0,1)}>C R \text { and } \mathrm{j} \neq \mathrm{rn}(\mathrm{i})\end{cases}$

The authors in [6] propose an eigenvector-based crossover operator, which utilizes eigenvectors of covariance matrix of individual solutions. Thus, the crossover is rotationally invariant. To avoid losing diversity of population, the offspring can be stochastically born from the parents with either the standard coordinate system or the rotated coordinate system. The authors in [6] also introduce a new parameter to control the probability of selecting one of the coordinate systems. They have shown that this scheme can increase the population diversity and prevent premature convergence. Additionally, another significant advantage of this operator is that it can be applied to any crossover strategy with minimal changes. Therefore, it can enhance any existing DE variant. The main idea is to exchange the information between the target vector and the mutant vector in the eigenvector basis instead of the natural basis. The covariance between $\mathrm{m}$-th and $n$-th dimension of the population in the G-th generation is given by

$$
\operatorname{cov}(m, n)=\frac{\sum_{i=1}^{N P}\left(x_{G, m, i}-\mu_{G, k}\right)\left(x_{G, n, i}-\mu_{G, n}\right)}{N P-1}
$$

where $\mu_{G, m}, \mu_{G, n}$ are the mean values of the variables in the $\mathrm{m}$-th and $\mathrm{n}$-th dimension respectively.

To compute the eigenvector basis we need to factorize the covariance matrix $\mathbf{C}_{G}=\left(c_{m n}, c_{n m}=\operatorname{cov}(m, n)\right)$ into a canonical form

$$
\mathbf{C}_{G}=\mathbf{Q}_{G} \Lambda_{G} \mathbf{Q}_{G}^{-1}
$$

where $\mathbf{Q}_{G}$ is the square matrix $(\mathrm{D} \times \mathrm{D})$ whose m-th column is the eigenvector $\bar{q}_{G, m}$ of $\mathbf{C}_{G}$, and $\Lambda_{G}$ is the diagonal matrix whose diagonal elements are the corresponding eigenvalues. The factorization of a matrix into a canonical form is called Eigen decomposition. The authors in [6] use the Jacobi's method [9] for Eigen decomposition. When the eigenvector basis is found, the i-th target vectors $\bar{x}_{G, i}$ can be expressed by $\left(\mathbf{Q}_{G} \bullet \bar{x}_{G, i}\right)$; the i-th mutant vectors $\bar{v}_{G+1, i}$ can be expressed by $\left(\mathbf{Q}_{G} \bullet \bar{v}_{G+1, i}\right)$. Then, a predefined crossover operator, such as binomial crossover, will exchange some of the elements of the mutant vector with some of the elements of its target vector to form a trial vector. The trial vector is then given by [6]

$$
\bar{u}_{G+1, i}=\left\{\begin{array}{l}
\mathbf{Q}_{G}^{*} \bullet \operatorname{xover}\left(\mathbf{Q}_{G} \bullet \bar{x}_{G, i}, \mathbf{Q}_{G} \bullet \bar{v}_{G+1, i}\right) \text { if } \text { rand }_{[0,1]} \leq P_{(10)} \\
\operatorname{xover}\left(\bar{x}_{G, i}, \bar{v}_{G+1, i}\right) \quad \text { otherwise }
\end{array}\right.
$$

where $\mathbf{Q}_{G}^{*}$ is the conjugate transpose of the eigenvector basis $\mathbf{Q}_{G}$, and $\operatorname{xover}(\bar{x}, \bar{y})$ is a crossover operator on two vectors $\bar{x}$ and $\bar{y}$, where $P$ is a new control parameter introduced in [6] which is called eigenvector ratio. Eigenvector ratio is between 0 and 1 and determines the ratio of the eigenvector based crossover operator and the other crossover operator. It must be noted that when $\mathrm{P}=1$ then only the eigenvector crossover operator is used, while when $\mathrm{P}=0$ then only the binary crossover operator is used. The main feature of this approach is that no matter how the crossover operator exchanges the elements in the eigenvector basis, the crossover behavior will become rotationally invariant in the natural basis.

\section{NUMERICAL RESUlTS}

We consider the 75 possible LTE base stations given in Fig. 1. Each one can be active (1) or not (0). If the base station is active then the range of the input power of the base station antenna is from 0 to $43 \mathrm{dBm}$ with a step of $1 \mathrm{dBm}$.

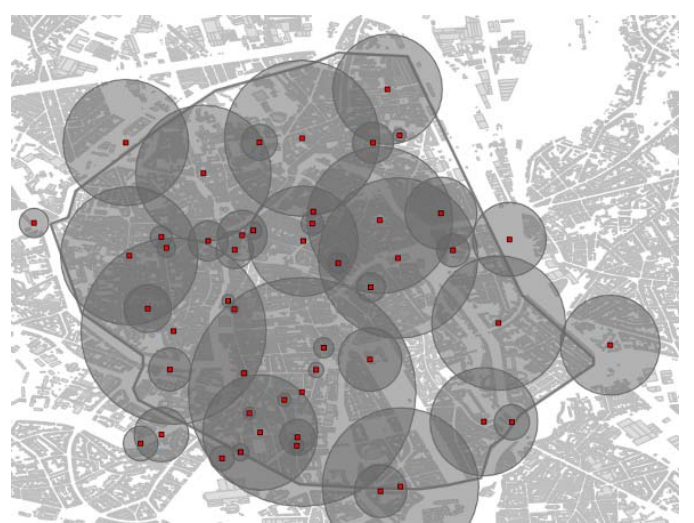

Fig. 2. Map of the city of Ghent with the active LTE base stations for the SISO case. The circles represent the coverage area of each base station.

The algorithm is executed 20 times. The results are compared. The DE-EIG algorithm is initialized with a population size of 100 and run for 1000 iterations. The first case is that of an LTE network without Multiple Input Multiple Output (MIMO). The total number of decision variables is $75 \mathrm{X} 2$ for this case (each base station can be active or not and have a value of input power).

The best-obtained result is that of a network with about $82.6 \%$ coverage and $48 \%$ power consumption (which means that the power consumption is $48 \%$ of the maximum power consumption assuming that all base stations are active and 
operate at maximum input power). The solution consists of 50 base stations. This solution is visualized in Fig. 2.

The second case is that of an LTE network supporting MIMO. In this case each base station could support MIMO and therefore an additional decision variable is required. The total number of unknowns is $75 \mathrm{X} 3$. The number of antennas is limited to $1 \mathrm{X} 1,2 \mathrm{X} 2,4 \mathrm{X} 4$ for both transmission and reception. DE-EIG has produced a network that has $95 \%$ coverage and $22.14 \%$ power consumption. The number of base stations in this network is 36 . Fig. 3 visualizes this case.

The final example is that of an LTE network supporting MIMO but with a different number of transmitting and receiving antennas. Therefore, the total number of unknowns increases to $75 \mathrm{X} 4$. The best-obtained result for this case is a network with $95 \%$ and $19 \%$ power consumption. This solution requires 31 LTE base stations. The network is shown in Fig.4.

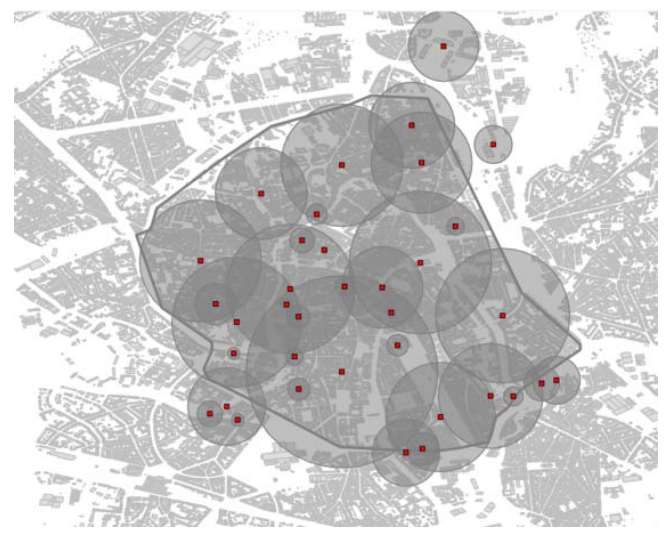

Fig. 3. Map of the city of Ghent with the active LTE base stations for the first MIMO case. The circles represent the coverage area of each base station.

TABLE II. COMPARATIVE RESULTS FOR THE THREE CASES OBTAINED BY DE-EIG

\begin{tabular}{|c|c|c|c|c|}
\hline Case & Best & Worst & Mean & $\begin{array}{c}\text { Std. } \\
\text { Dev. }\end{array}$ \\
\hline SISO & -310.05 & -259.59 & -290.58 & 15.06 \\
\hline MIMO case 1 & -484.32 & -461.82 & -476.63 & 7.6 \\
\hline MIMO case 2 & -500.33 & -473.17 & -483.81 & 8.12 \\
\hline
\end{tabular}

Table II summarizes the obtained results for the three cases by the DE-EIG algorithm. We notice that as expected the value of the objective function increases in the MIMO cases. The MIMO cases present lower standard deviation values than the SISO case. The best objective function value is that of the second MIMO case.

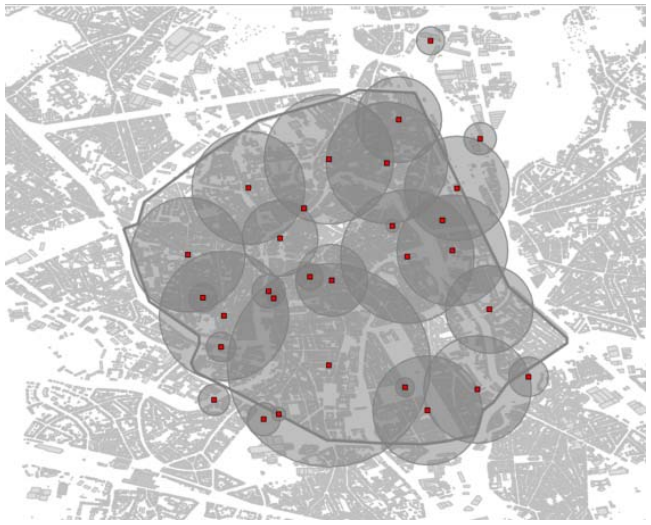

Fig. 4. Map of the city of Ghent with the active LTE base stations for the second MIMO case. The circles represent the coverage area of each base station.

\section{CONCLUSION}

The problem of designing LTE networks for optimal coverage with the lowest power consumption is addressed in this paper. We have presented a novel design approach based on Differential Evolution with Eigenvector-based crossover operation. The numerical results that we have shown have proven the effectiveness of this approach

\section{REFERENCES}

[1] W. Van Heddeghem, S. Lambert, B. Lannoo, D. Colle, M. Pickavet, P. Demeester, Trends in worldwide ICT electricity consumption from 2007 to 2012, Computer Communications, 50 (2014) 64-76.

[2] M. Deruyck, W. Joseph, E. Tanghe, L. Martens, Reducing the power consumption in LTE-Advanced wireless access networks by a capacity based deployment tool, Radio Science, 49 (2014) 777-787.

[3] M. Deruyck, W. Vereecken, W. Joseph, B. Lannoo, M. Pickavet, L. Martens, Reducing the power consumption in wire-less access networks: Overview and recommendations, Progress In Electromagnetics Research, 132 (2012) 255-274.

[4] R. Storn, K. Price, Differential evolution - A simple and efficient heuristic for global optimization over continuous spaces, Journal of Global Optimization, 11 (1997) 341-359.

[5] E. Mezura-Montes, J. Velazquez-Reyes, C.A. Coello Coello, A comparative study of differential evolution variants for global optimization, in: GECCO 2006 - Genetic and Evolutionary Computation Conference, Seattle, WA, 2006, pp. 485-492.

[6] S.M. Guo, C.C. Yang, Enhancing differential evolution utilizing eigenvector-based crossover operator, IEEE Transactions on Evolutionary Computation, 19 (2015) 31-49.

[7] M. Deruyck, E. Tanghe, W. Joseph, L. Martens, Modelling and optimization of power consumption in wireless access networks, Computer Communications, 34 (2011) 2036-2046.

[8] R. Storn, Differential evolution research - Trends and open questions, Studies in Computational Intelligence, 143 (2008) 1-31.

[9] J. Demmel, K. Veselić, Jacobi's method is more accurate than QR, SIAM J. Matrix Anal. Appl., 13 (1992) 1204-1245. 